\title{
Thermoplastic J Integral Calculation for a Semi-Elliptical Crack in the Railway Wheel
}

\author{
${ }^{1}$ Azadeh Haidari, ${ }^{2}$ Mojtaba Heidari \\ ${ }^{1}$ Center of excellence in railway transportation, Iran University of Science and Technology, \\ Tehran, Iran. \\ ${ }^{2}$ College of mechanical engineering, Isfahan University of technology. Iran, Isfahan
}

\begin{abstract}
This paper provides a method for addressing surface crack growth in railway wheels, taking into account rail and brake blocks effects. finite element analysis (FEA) using the J-integral crack tip parameter has been employed to study 3-D semi elliptical crack growth in the railway wheel with strain hardening property, subjected to fatigue loading due to axle load, rail-wheel contact forces, motive torque, brake blocks pressure, friction and its resulting thermal loads of braking. For this purpose, a FE model of a wheel, with two brake shoes and a portion of rail are created and realistic loads and boundary conditions, which may correctly model the rolling and stopping procedure, are applied to the railway wheel model. It is assumed that the wheel has contained a surface semi-elliptical crack on its tread surface. The importance of axle load, speed, plasticity and braking thermal load effects on growing of a surface crake situated on a thread of wheels are demonstrated in this paper.
\end{abstract}

Keywords: elastic-plastic, hardening, crack, thermo-mechanical

\section{INTRODUCTION}

The sudden failure of equipment and constructions, not only cause serious physical consequences, but also economic losses can be significant. The major source of failure of structural components is fatigue crack growth. In the past, the $\mathrm{S}-\mathrm{N}$ curves were the only engineering tools, and crack propagation was not considered to predict lifetime. Nevertheless, at present, studying crack growth behavior has been made possible by the linear elastic fracture mechanics (LEFM) or elastic-plastic fracture mechanics (EPFM) and the prediction of remaining lifetime of components is accessible. This is of great practical importance in order to know if a part can be still used or must be replaced

Observations on the railway wheel indicate there are different types of damage able to cause deterioration or catastrophic failure of them. One of the most common ones is thermal cracking due to braking thermal loads. By limiting the interest to the damage process related to rolling contact fatigue we can distinguish surface contact fatigue, that consists in the initiation and propagation of a crack originated on the free surface due to the cyclic repetition of the contact pressure and thermal loads created at braking cycles, and sub-surface contact fatigue, that consists in the initiation and propagation of a crack originated some millimeters under the free surface and is promoted by the presence of microscopic material defects. I this study due to considering braking cycles and their effects on generating high temperature, a semi-elliptical surface crack is considered [1].

Based on linear elastic fracture mechanics, fracture and stable crack growth occurs when the stress intensity factors reach to a critical value. This theory is based on the assumption that the material is perfectly elastic and brittle as a result of the effect of plastic deformation at the crack tip is too small regardless and thus during crack growth, there is no energy consumption for creation or expansion of plastic zone around the crack tip. But for ductile materials like metals, before occurring the final fracture, Substantial plastic deformation occurs in the body and therefore the size of plastic zone around crack tip can't be ignored. For these kinds of problems, LEFM is not valid and another criterion (EPFM) should be used.

EPFM had its beginnings in 1961, when Wells [2] noticed that initially sharp cracks in high toughness materials were blunted by plastic deformation. Wells proposed that the distance between the crack faces at the deformed tip be used as a measure of fracture toughness. The stretch between the crack 
faces at the blunted tip is known as the crack tip opening displacement (CTOD). In 1968 [3] developed another EPFM parameter called the $\mathrm{J}$ integral by idealizing the elastic-plastic deformation around the crack tip to be nonlinear elastic.

Our study is carried out through numerical simulations. The current study incorporates an elastoplastic material response, which increases computational costs. Analyses of surface cracks subjected to (frictional) rolling contact and braking thermal loading rarely found in the literature. The majority of the existing analyses are restricted to elastic material properties with two-dimensional contact or three-dimensional (3D) load conditions typically under the presumption of Hertzian contact conditions, see e.g. [4]. The characteristic of this study is modeling of the crack and quantify it for determining effects of thermal loads of braking and plastic strains induced from cyclic hardening property of the material on the crack behavior while in the vast majority of previous studies crack was not modeled and no proper quantity provided for revealing the role of crack existing on the life of the structure. As another simplification used in previous studies, is the use of 2D Green functions to translate a 3D stress field to stress intensity factors, see [4].

\section{THERMAL LOADS OF BRAKING}

Thermal loading of a railway wheel is a consequence of frictional wheel-rail, and/or wheel-brake shoe contact. Extreme thermal loading (e.g. as a consequence of malfunctioning brakes) may lead to catastrophic wheel failures.

positive (with respect to the rolling direction) traction corresponding to an accelerating wheel was found to give higher magnitudes of equivalent von Mises stress and equivalent plastic strain in the bulk material. In comparison, as shown in Fig. 1, a negative traction (i.e. braking of the wheel) will induce higher longitudinal stresses. In particular brake loads introduce a tensile residual stress which will promote subsequent crack growth.

Braking process is simulated by pressing two brake blocks to the tread surface of the wheel with initial angular velocity of $50 \mathrm{rad} / \mathrm{s}$ during one revolution of the wheel with high retardation of $75 \mathrm{~m} / \mathrm{s}^{2}$. In this case a thermal power of $500 \mathrm{KW}$ is generated in wheel-brake shoe contact region. On the other hand in rail-wheel contact zone, the power of $380 \mathrm{KW}$ generated $\left(\mathrm{Hi}=1 / 2 \mathrm{~m}_{\text {wheel }} \mathrm{rv}\right.$, with $\mathrm{m}_{\text {wheel }}=12500$ $\mathrm{kg}$ for an axle load of 25 tonnes). The thermal simulations account for cooling by radiation and convection calibrated using results from field tests [5]

The most important temperatures are those obtained at the interface of the sliding surfaces,but knowledge of the temperatures below the surfaces, is needed for calculating the thermal stresses. These temperatures can be obtained from the output of Abaqus software in the thermal analysis of the model. Figure 1 shows the

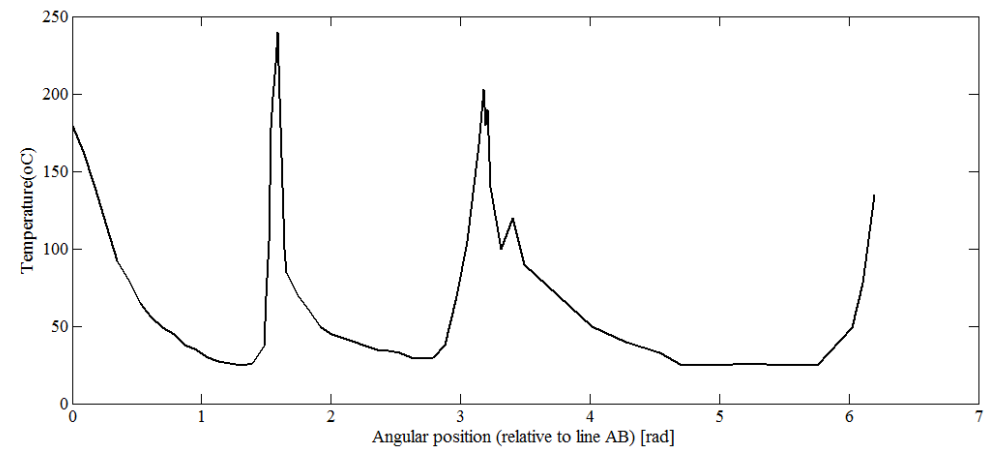

Fig1.Temperature variation of the crack tip (point 1) during one revolution in braking step

As can be deduced from this figure, in the contact regions of wheel with rail and two left and right brake blocks, sudden and significant temperature rise generated. According to our model, because taking into account the coefficient of thermal expansion for all components, theses temperature variations, create high thermal stresses and strains.

The order of thermal strains and stresses are in order of the corresponding values of rolling contact loads and this is emphasizing that for the wheels, effects of braking thermal loads are non-negligible. On the other hand, our studies indicated the importance of hot bands and/or hot spots which develop within the contact regions and they may lead to a highly non-uniform temperature concentration and 
high thermal strains and stresses [6]. That is why in this paper thermal loads of braking are considered by modeling of two brake blocks.

\section{Strain Hardening Equation}

Based of experimental observations, the repeated rolling and sliding contact of rail-wheel and wheelbrake shoes which exceed the shakedown limit, cyclic plastic deformation of the wheel in the near surface layers occurs with every cycle of the loading. In order to analyze this process in a realistic way, it is necessary to use a cyclic hardening law which incorporates the essential features of the material behavior. Most previous analyses of the problem have assumed either that the material was perfectly plastic with no hardening or linear kinematic hardening. There are serious drawbacks to both of these models. The assumption of perfect plasticity greatly overestimates the plastic strains observed in practice, particularly with steel, and leads to difficulties in the analysis of surface shear which occurs under high tractive force. The linear kinematic hardening model excludes the possibility of steady accumulation of plastic strain, known as ratcheting, which is a feature of many practical and experimental rolling and sliding contacts.

Our previous study shows that differences between linear elastic and elastic-plastic responses for the semi-elliptical surface crack is considerable and therefore, it can be noted how the hardening model is able to capture the accumulation of plastic strain and changes the fracture life of wheel [7]. By considering plasticity, using the Hertz contact theory gives results with less precision since according to this theory, it is assumed that the materials at contact are elastic while experimental and also our FE results imply on the non-negligible plastic deformation at contact surfaces and therefore for our elastic-plastic model, modeling of rail and brake blocks is more important [8]. On the other hand, for material with considerable plastic strains the commonly known LEFM method can't be used and it is necessary to use fracture mechanics methods that can consider the material plasticity (EPFM) which will be explained in the next section. Due to cyclic loading of the wheel during its operation and nonnegligible plastic strains induced from the cyclic hardening property of the wheels steel, in this paper cyclic hardening property by using the plots of stress-strain of railway wheel steel during different cycles of loading is considered.

Plots of $\Delta \sigma$ versus $\Delta \varepsilon$ were obtained from the low cycle fatigue experiments is shown in figure 2 [9].

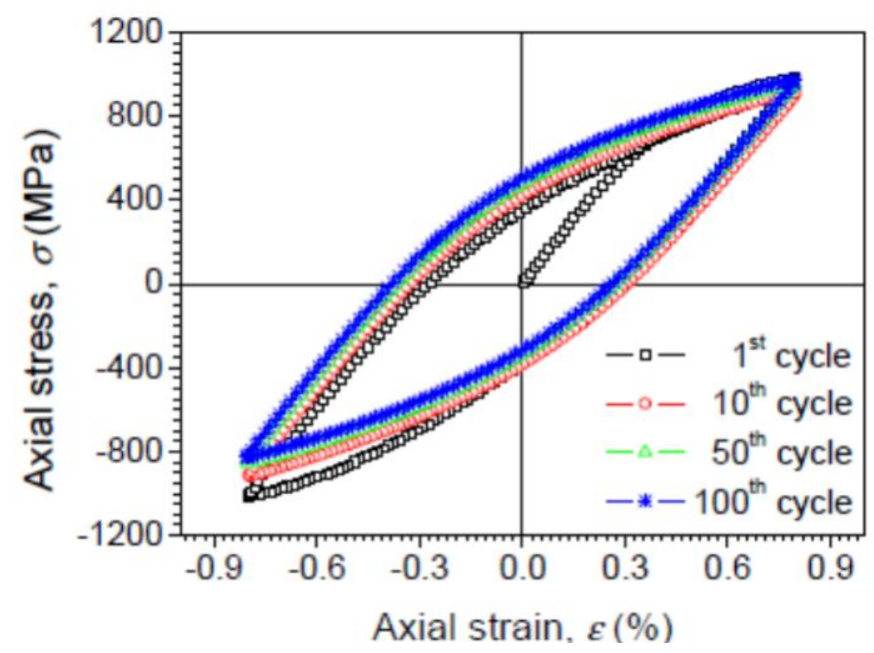

Fig2. Cyclic hysteresis loops under uniaxial symmetrical strain cycling [9].

As can be seen from this figure, the slope of the stress strain curve during a positive stress increment is lower than that during a negative stress increment. Thus, the material will accumulate strain in the direction of the mean stress, as shown in Figure 3[10]

Simulation of cyclic hardening property is done in Abaqus 6.14.2 software in property module.

\section{J-INTEGRAL METHOD}

Based on fracture mechanics, the crack growth rate, depending of the applied loads and material property, is dependent of one of parameter such as crack tip opening displacement, stress intensity factor, energy release rate, ... which among them, $\mathrm{J}$ integral has the wider application range and can be used as a proper basis for crack analyses 
Fracture mechanics science has two main approaches for crack effects determination: Linear elastic fracture mechanics (LEFM) and Elastic-Plastic fracture mechanics (EPFM). In LEFM the local stresses in vicinity of crack tip, analyze according to elasticity theorem, used for studying the crack growth. This method is applicable only until the plastic zone at the crack tip is small enough in comparison to characteristic sizes of the crack; otherwise, LEFM becomes non-applicable and EPFM should be used. In this case instead of stress intensity factor of crack, other methods like J integral method is used for crack behavior and growth studying.

The results of previous research show that using ABAQUS software, the energetic approach reveals a good efficiency with great practical simplifications since no singular element and orthogonal mesh are required. Moreover, the J-integral method shows some advantages compared to the displacement extrapolation one. First of all, this method may be applied automatically with the ABAQUS code. Then, the knowledge of the exact displacement field in the vicinity of the crack tip is not required and the use of singular finite elements is not essential anymore. Besides, non-orthogonal meshes are without effect on the SIF calculations. The user has just to be sure that a convergent value is obtained on the different rings. As a consequence, this approach seems to be particularly suitable to deal with the fatigue growth of general Cracks.[11]

Many authors apply the stress intensity factors (SIFs) as parameters for determining crack effects. These parameters calculate just on the first node on the crack face and by using fine meshes consider that the distance $r$ is very close to 0 . However, this method may be quite difficult and costly to use. Indeed, the kind of elements need for calculating SIFs is not always in the library of the finite element codes. ANSYS has this possibility [12] but ABAQUS, which is used in this paper, has not. The user must, manually, collapse the finite elements and relocate the mid-side nodes. This task may be easy in 2D. Unfortunately, with 3D models, when the crack front is curved, like, the semi-elliptical crack modeled in this study, and subjected to great changes during the fatigue growth process, lots of problems occur. First of all, this method requires necessarily an orthogonal mesh in the vicinity of the crack [13]. Secondly, SIFs values only can use in problems which the radius of plastic zone around the crack, is small compared to the crack size and can't be used in elastic-plastic materials. Taking these difficulties into account, it could be interesting to study other possibilities to obtain the other crack intensity parameter distribution. One of the methods reported in the literature is an energetic one. It consists in determining the J-integral values. The J-integral values can be obtained quite far from the crack tip. Fine meshes and collapsed quarter-point finite elements are not necessarily required in order to exactly simulate the singularity. Besides, non-orthogonal meshes have no effect on the results in this method [14].

By comparing different methods and due to importance of crack tip plastic zone size in determination of crack growth criterion and also high plasticity caused by cyclic hardening properties of R7T steel of railway wheel, in this paper $\mathrm{J}$ integral approach is used for crack estimation.

As an approximate maximum plastic size of the crack tip which is equal to $1 / 8$ crack length, the contours chosen for determination of $\mathrm{J}$ integral, should be out of this distance and therefore in meshing the crack front region, this point, should be considered.

In order to obtaining the more accurate and reliable results, the appropriate number of contours must be selected to ensure that the regions far enough away from high singular stresses of crack around, are selected. In this article 10 numbers of contours are considered around the crack[15].

Structural strain-hardening materials can be subject to elastic-plastic loading during overloads in application. Thus, an analysis to determine the stress intensity around semi-elliptical cracks undergoing elastic-plastic loading is important in understanding the structural integrity of components in these conditions. Because the J-integral consists of energy type terms within a relatively remote boundary encompassing the crack tip, it is not necessary to use elements with nodes at the quarter points of the edges to account for the stress singularity at the crack tip [14]

Calculations of $\mathbf{J}$ integral in this paper is performed with the finite element code ABAQUS. Provided that the user has defined the nodes of the crack front, the software automatically finds the contours in order to carry out the energetic analysis and then returns a SIF value for each contour. This integral is theoretically path independent. However, it is widely accepted that the first contour does not provide good results because of numerical singularities [15]. Therefore, the SIFs have been obtained from the second contour or even further from the crack tip, in order to get a convergent value. The results are discussed in next section. 


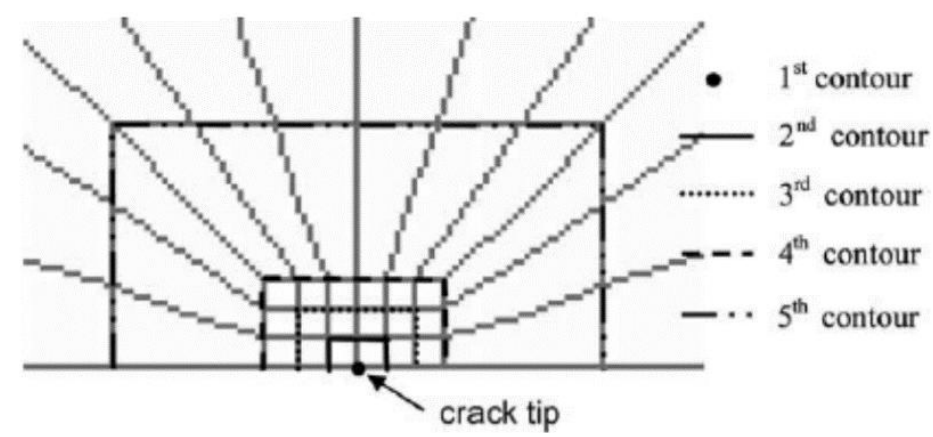

Fig3. Various contours for the calculation of J-integral with 3D coarse mesh,

\section{Modeling}

A finite element analysis (FEA) using the J-integral crack tip parameter has been employed to study 3-D semi elliptical surface crack in the railway wheel with cyclic hardening property. This wheel is subjected to angular velocity of $50 \mathrm{rad} / \mathrm{s}$, axle load of $145 \mathrm{KN}$ and rail-wheel contact shear and normal stresses in the first step of modeling. While in the second step, two cast iron brake shoes compress to the wheel tread surface with braking pressure of $700 \mathrm{MPa}$ and generate high friction in the contact zones and also cause to occurrence of slip of the wheel on the rail surface. By considering thermal properties of wheel, rail and brake shoes and applying thermal degree of freedom for components, the temperature increase of the wheel caused by the braking process can be achieved.

Figure 4 shows the whole model include wheel, rail and brake blocks and a schematic view of the semi-elliptical crack considered in the wheel tread surface.
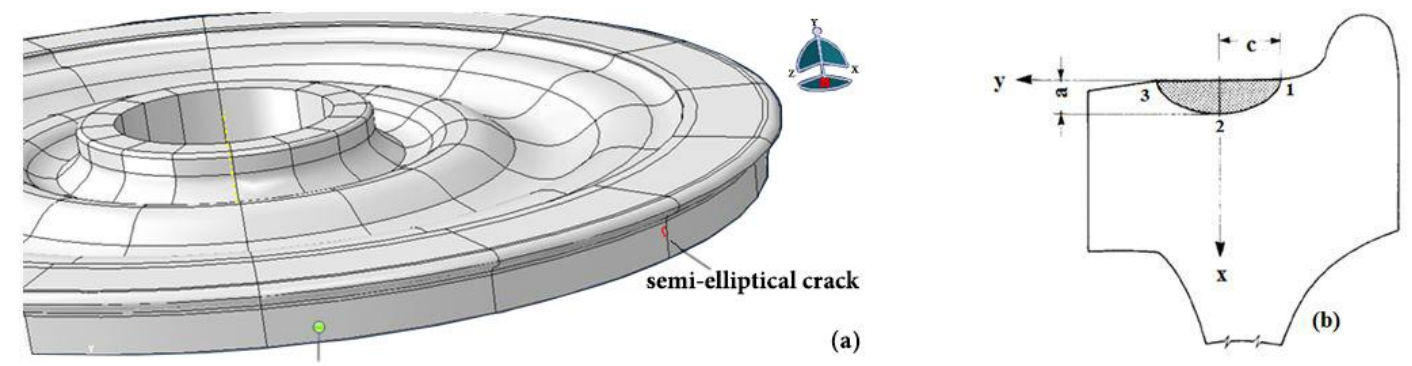

Fig4. (a) 3D model of cracked railway wheel, (b) 2D schematic view of semi-elliptical crack.

The mechanical and thermal properties of all components are chosen based on existing experimental values of references.

Wheel axle load and boundary conditions are considered by defining a reference point in the center of the wheel and rigid connection of this point with the internal surface of wheel. axleload of $145 \mathrm{KN}$ is applied to the reference point. The rotational speed $(50 \mathrm{rad} / \mathrm{s})$ in the same point is considered for the wheel

too.

In addition to this, under the assumption of constant speed, a torque (due to wheel speed) in the ydirection is applied to the reference point. Linear displacement degrees of freedom of the wheel in two directions of $\mathrm{y}$ and $\mathrm{z}$ are completely constrained but in $\mathrm{x}$ direction it can move. For two sides of rail section the clamped boundary condition is applied. The shoes are also constrained from all rotation and displacement DOFs except for the axis perpendicular to their contact region. A convection factor of $100 \mathrm{~W} / \mathrm{m}^{2} .{ }^{\circ} \mathrm{c}$ is considered for the wheel [16].

A coarse mesh is used for the wheel hub and web whereas a finer mesh is introduced in the vicinity of the wheel-rail and wheel-brake blocks contact regions and in particular in the vicinity of the crack. Parts with incompatible meshes are connected with surface-based tied constraints. Simulations are carried out in Abaqus, version 6.14-2[17].

\section{Mesh Sensitivity}

To determine the appropriate size of elements, the mesh sensitivity analysis is used for our model and the results of two different element size ranges are compared.

As a characteristic mesh size, the element lengths on the crack faces for the two mesh used in this paper are given in Table 1 and their corresponding values of von Mises stresses are listed Mesh 
sensitivity results with respect to opening and shear displacements of the crack tip (point 1 in Fig 4b are presented in Fig. 5. As can be seen from the convergence of the results, it may be concluded the meshing and element sizes used in this study are proper for determining the correct response and there is no need to use the finer mesh, which leads to increasing the analysis time and access memory of the computer, therefore mesh 1 is sufficiently fine and it will be employed in subsequent analyses.

Table1. Mesh data for analysis of our model.

\begin{tabular}{|l|l|l|l|}
\hline & Minimum and maximum element size (mm) & Number of elements & Max von Mises stress \\
\hline Mesh 1 & $0.3-5$ & 25488 & $619 \mathrm{MPa}$ \\
\hline Mesh 2 & $0.1-3$ & 41265 & $641 \mathrm{MPa}$ \\
\hline
\end{tabular}

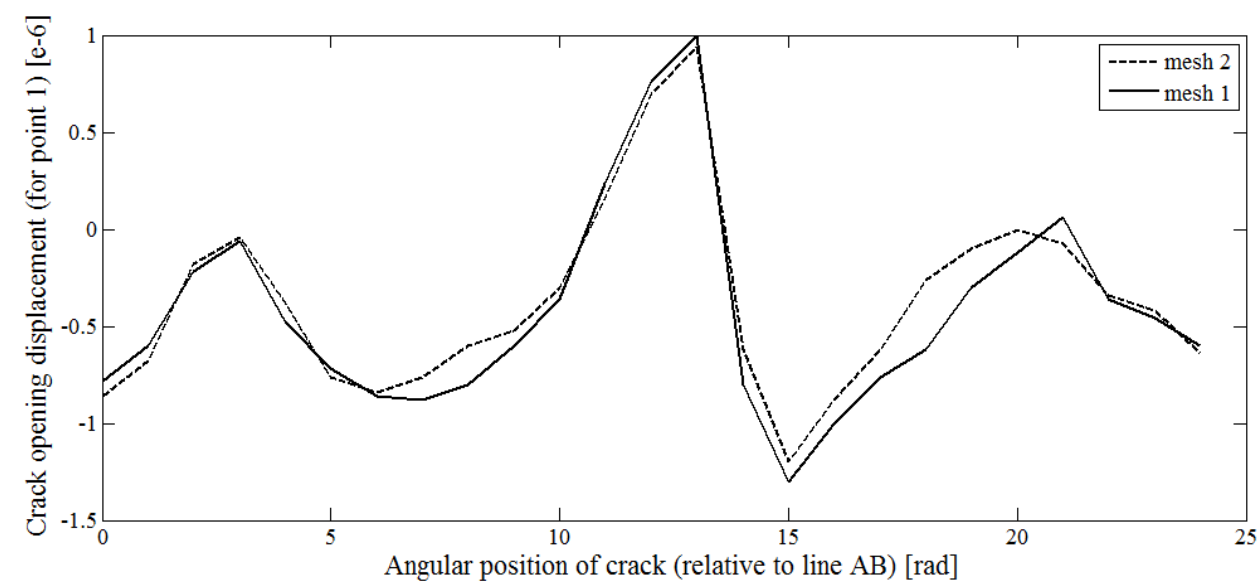

Fig5. Crack opening displacement for dirctcrcak positions.

As can be seen from this figure when the crack moves toward the contact regions (rail-wheel and wheel-shoes contact areas), first the opening mode displacement and therefore mode I of stress intensity factor (KI) increases and reaches to its maximum value in the center of contact nearly and then, by moving the wheels further, opening displacement of the crack (and also KI) dramatically decreases. Switching the signs of CTD from positive to negative or Vic versa means that the crack flank deformation changes from opening to closing mode and Vice versa. [18]The trend observed for variation of opening crack tip displacement can be found for its shear displacement as well However, the place of maximum values occurrence for opening and shear displacements are a little different.

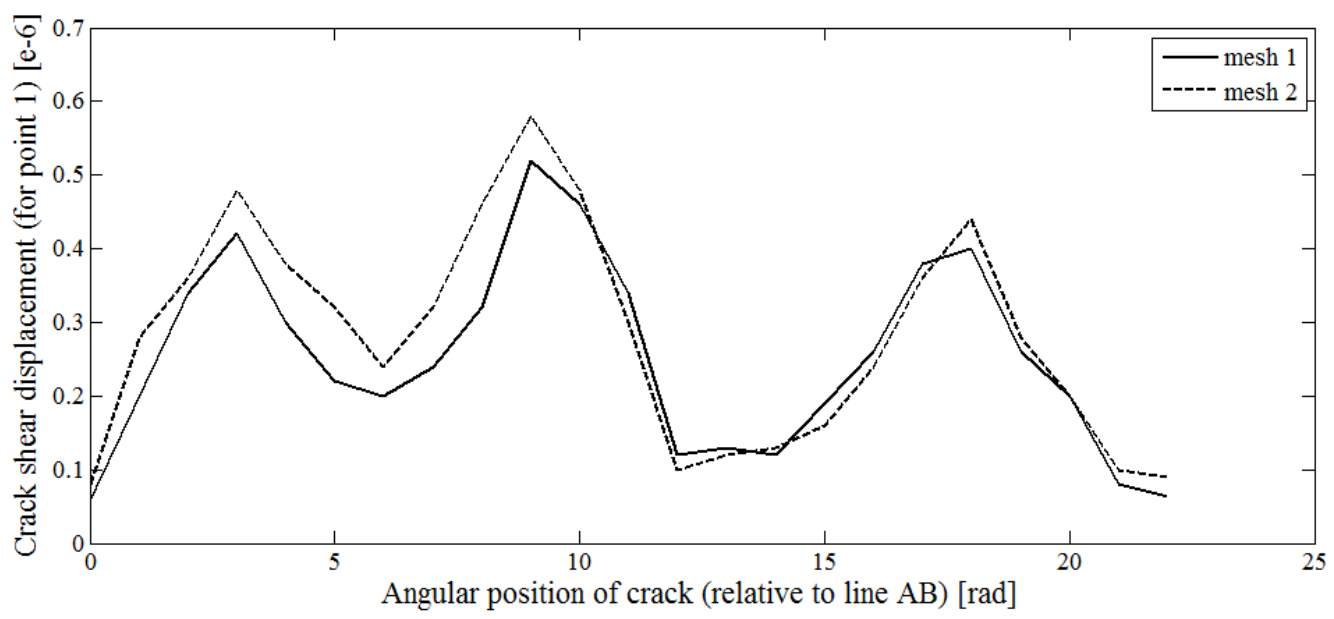

Fig6. Crack shear displacement in crack positions

\section{RESULTS AND DISCUSSION}

Rail, wheel and brake shoes are meshed with C3D8R elements. Von Mises stress field of the wheel is shown in Fig.7. As can be seen, maximum value of this stress is 619MPa and occurs on the crack tip. This value of stress is indicative of plastic deformation of the wheel in the contact area and so this is good reason to inefficiency of Hertz contact theory in the contact region because the Hertz theorem, it is assumed that materials in contact zone, remain elastic. The plasticity, cause reduction of maximum compressive stress and increase the strain components and contact area. 


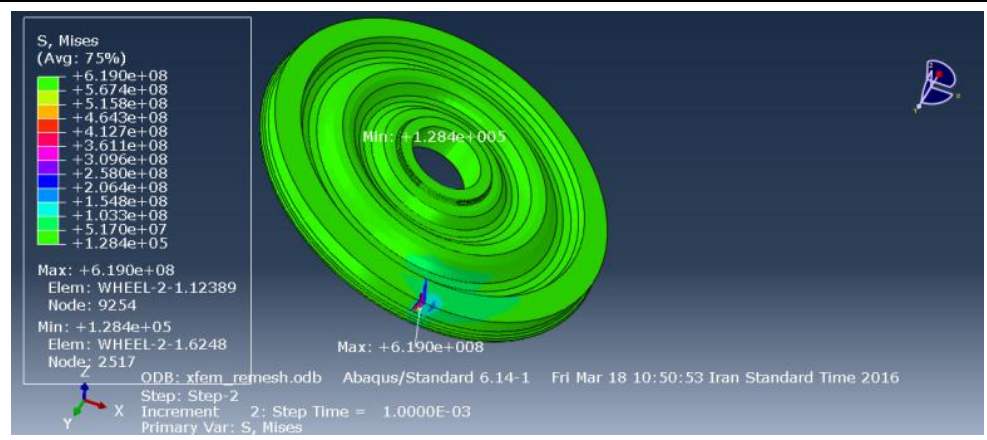

Fig7. Von Mises stress field of the wheel

Using Abaqus software calculation, minor and major axis of the contact ellipse are 5 and $8 \mathrm{~mm}$ respectively which are larger than the sizes obtained by Hertz theory therefore in the contact region in addition to decreasing the stress, relatively high strain accumulation is obtained. [19]

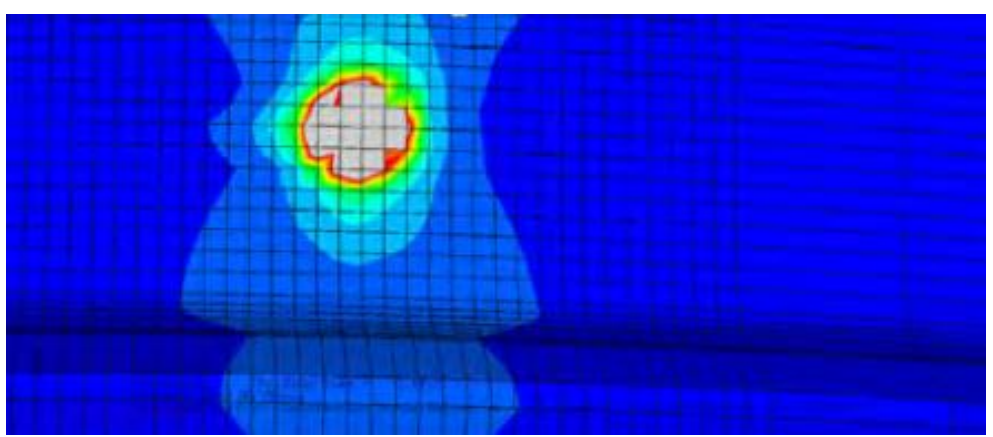

Fig8. Rail-wheel contact region

The values of $\mathrm{J}$ integral obtained from FE results for 10 contour considered in the model, are shown in Fig .9 In practice, ABAQUS provides evaluation of the $J$-integral on as many contours as the user requests. The first contour is the ring of elements immediately adjacent to the crack front, and subsequent contours are generated moving away from the crack border. Since the $J$-integral should be path independent, the variation of $J$ among the contours enough far away from the crack tip, can be interpreted as an indicator of mesh quality.

Finite element results for the cracked wheel are shown for 10 contours around the semi-elliptical crack. As can be seen, the results from Contours 6 through 10 are acceptably close, whereas the results from the Contours closer to crack borders tend to oscillate. According to the ABAQUS Benchmarks Manual, the calculations from these contours (inner contours) can be ignored because of numerical inaccuracies in the stresses and strains at the crack fronts and their nearby areas. Moreover, the consistency of results from contours far away from the crack (outer contours) suggests that the finite element mesh is acceptable for the task at hand. [20]

This figure is indicative of path independency of the obtained $\mathrm{J}$ integral from contours 6-10 nearly. As can be seen, the rang of difference for the first 5 contours is rather remarkable while for contours farther from the crack tip, the $\mathrm{J}$ integral values for different contours becomes smaller so that the corresponding values of contours 9 and 10 which are enough far from the crack tip and its plastic zone radius are approximately the same.

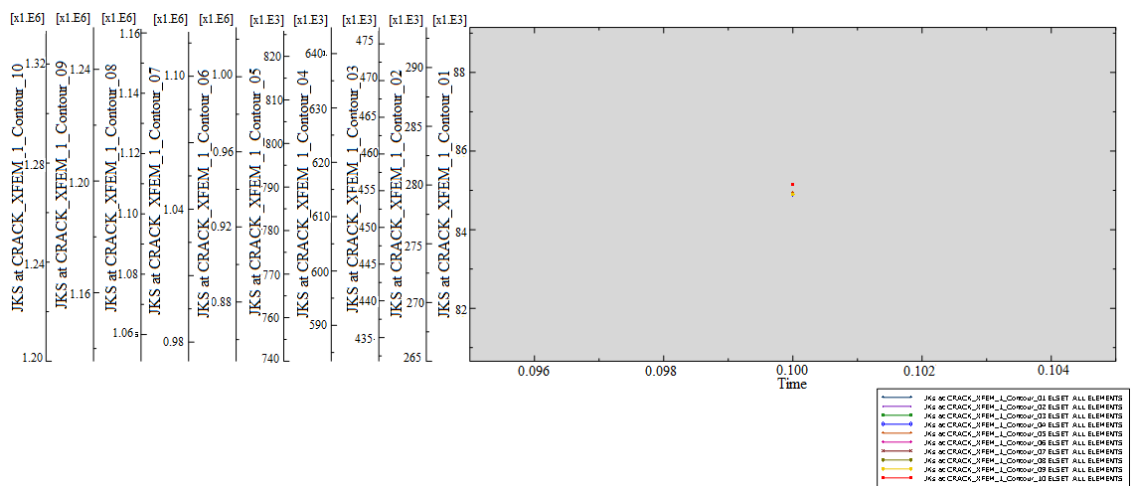

Fig9. J integral values for different contours around the crack 
The corresponding values of stress intensity factors of crack tip 1 for 10 different contours considered in our model are illustrated in Fig. 9.
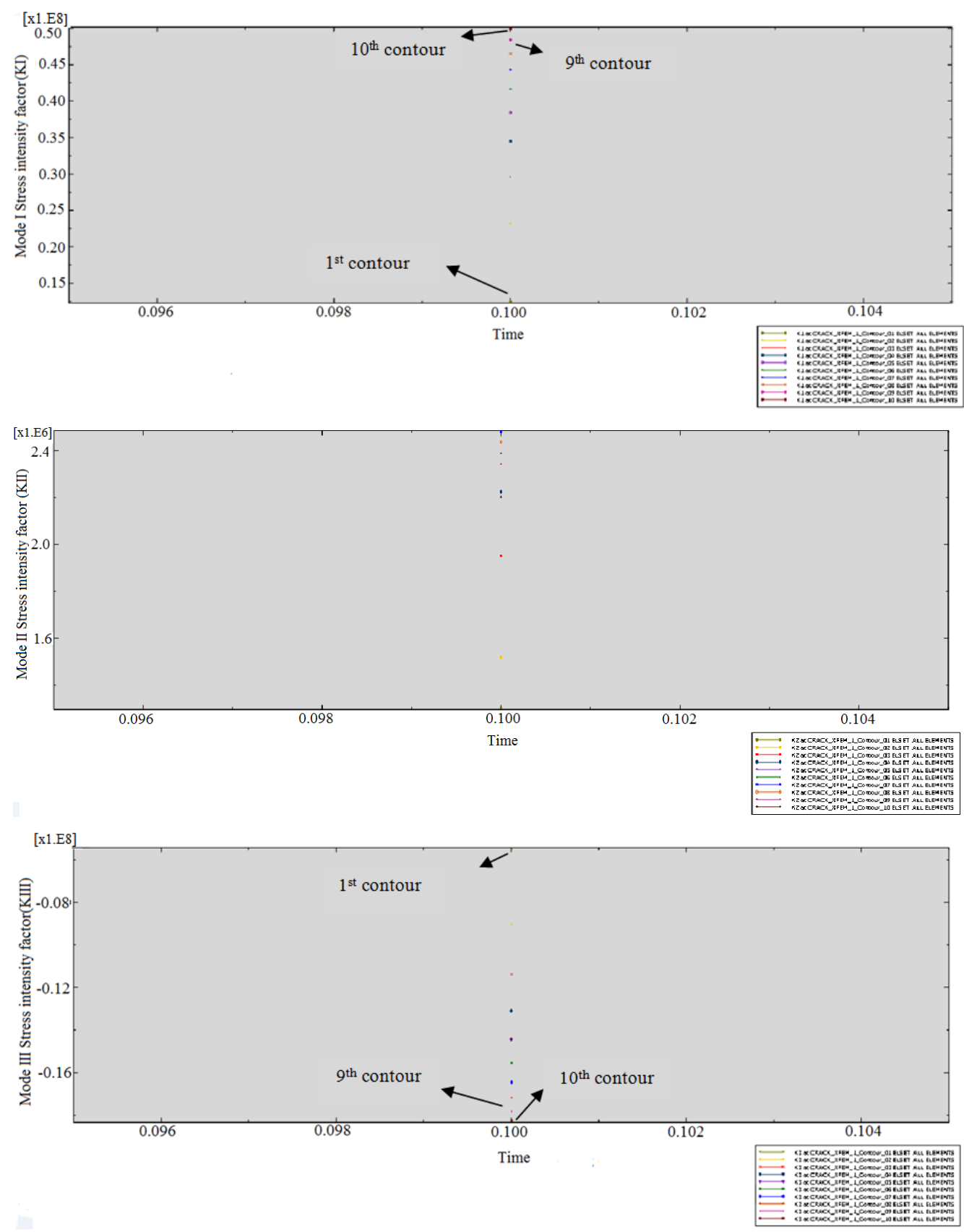

Fig10. Mode I, II and III stress intensity factor for different contours

In this figure as the previous figure the range of variation for the contours closer to the crack faces is considerable and by getting away from the crack tip, the values become closer. These figures confirm the values of contours are farther from the crack borders nearly coincide especially for contour 9 and 10.

By considering stress intensity factor components obtained from output of software and placing them in the following equation presenting by Liu et.al. The effective stress intensity factor can obtained.

$K_{\text {mixed }, e q}=\frac{1}{B} \sqrt{\left(K_{I}^{2}+\left(\frac{K_{I I}}{s}\right)^{2}+\left(\frac{K_{I I I}}{s}\right)^{2}+A\left(\frac{K^{H}}{s}\right)^{2}\right)}$

In this equation, material parameter s related to the material ductility and the value of it, is assumed to be equal to 0.6 for rail wheel steel used in this article. The superscript $\mathrm{H}$ indicates the hydrostatic stress related term. . " $A$ " and " $B$ " are material parameters that are listed in Table 2. 
In the conditions of small amount of crack plasticity area (for elastic problems), $\mathrm{J}$ integral and stress intensity factors are linked by the following equation:

$$
\Delta J=\frac{(\Delta K)^{2}}{E^{\prime}}
$$

Which $E^{\prime}=E /(1-v 2)$ is plane strain elastic module. For elasto-plastic problems with non-negligible plastic strains, the following relationship is established between $\mathrm{J}$ integral and stress intensity factor:

$$
\begin{aligned}
& \Delta J_{\text {total }}=\Delta J_{\mathrm{el}}+\Delta J_{\mathrm{pl}}=\frac{(\Delta K)^{-}}{E^{\prime}}+\frac{\eta A_{\mathrm{pl}}}{B b_{0}} \\
& b_{0}=W-a_{0} \\
& \eta=2+0.522 \frac{b_{0}}{W}
\end{aligned}
$$

In this equation, Jel and Jpl are elastic and plastic parts of $\mathrm{J}$ integral. By putting the obtained values for KI, KII and KIII and calculating $\Delta$ Keff, the elastic part of $\mathrm{J}$ integral is obtained equal to about $15 \mathrm{KJ} / \mathrm{m} 2$ while the total value of $\mathrm{J}$ integral is about $1200 \mathrm{KJ} / \mathrm{m} 2$ and is considerably higher than elastic part. Apl is plastic area under the force-displacement curve and $\mathrm{W}$ and $\mathrm{B}$ are the specimen width and depth respectively. In this equation $a_{0}$ is a symbol of crack size and $b_{0}$ indicative of un-cracked ligament of the specimen.

The values of $\mathbf{J}$ integral shown in Fig9, represent the total values of $\mathbf{J}$ integral which are related to proportion of all parameters considered in the model such as: elastic, plastic and thermal strains. The contribution of elastic part on the J-values obtained in this section. In the following, the share of thermal strains on the results will be explained.

\section{EFFECT OF THERMal STRAins}

For obtaining thermal strain effects on the $\mathrm{J}$ integral value for semi-elliptical crack modeled in this study, analyses are done once without considering thermal load (mechanical analysis) and once again by considering its effect (thermo-mechanical analysis). Therefore thermal strain effect can be obtained. By calculating difference between these two analyses. Results of $\mathbf{J}$ integral values for thermo-mechanical and mechanical analyses are shown in Table 2

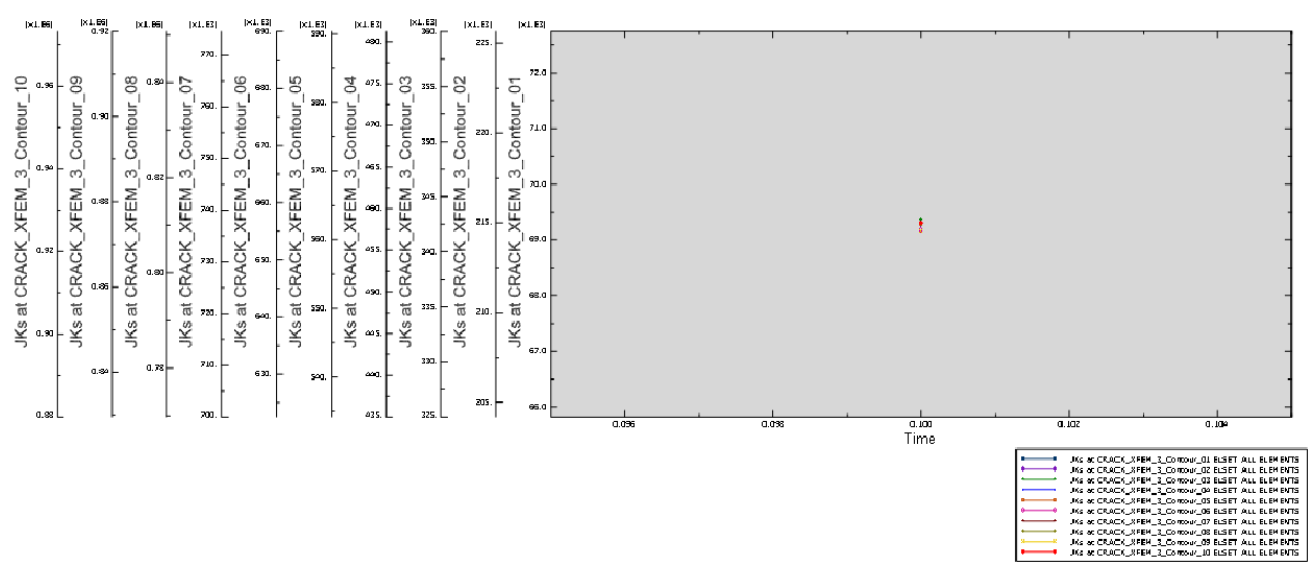

Fig11. J integral values for various contour by consideration thermal loads of braking.

Table2. Mechanical and thermo mechanical results for 6-10 contours

\begin{tabular}{|l|l|l|l|l|l|}
\hline & Contour 6 & Contour 7 & Contour 8 & Contour 9 & Contour 10 \\
\hline Thermo-mechanical analysis & 940 & 1004 & 1100 & 1120 & 1127 \\
\hline Mechanical analysis & 655 & 735 & 810 & 875 & 900 \\
\hline
\end{tabular}

By comparing results shown in this table the mean value of $\mathrm{J}$ integral for mechanical analysis is about $795 \mathrm{KJ} / \mathrm{m}^{2}$ and this value for thermo-mechanical analysis is nearly $1057 \mathrm{KJ} / \mathrm{m}^{2}$.

Difference between the results of analyses shows the importance and crucial role of thermal strains created during braking on the crack behavior of the wheels. 
The results show that the amount of thermal $\mathbf{J}$ integral is about $33 \%$ of mechanical value which is non-negligible.

\section{CONClusion}

The significant contribution of the all three crack growth modes which has been observed in this research for a semi-elliptical crack in the wheel is one of the distinction tips of this article. In almost all previous researchers the cracks had studied only by a two-dimensional crack model which is not able to detect mixed mode crack deformation and crack propagation had investigated by calculating stress intensity factors regardless of the crack tip plastic zone while due to hardening characteristics and high levels of plastic deformations, ignoring the effect of crack tip plasticity, does not give correct answers. Therefore in this article, instead of calculating the stress intensity factors ( $\mathrm{K}$ factors), the energy based method of $\mathrm{J}$ integral is used.

One advantage of this method is that the cumulative damage induced from the complex history of stresses and strains due to rolling contact and brake pressure loads can investigate by considering the plastic and thermal strains and hence the more reliable results is obtained. The $\mathrm{J}$ integral method has the ability of correct prediction of crack growth without shortcomings and disadvantages of the $\mathrm{K}$ factor method. By using this method, we can consider the high plastic deformations due to cyclic hardening property of the wheel steel and therefore the higher accuracy can be obtained.

\section{REFERENCE}

[1] M. Guaglianoa, M. Paub ,"An experimental-numerical approach for the analysis of internally cracked railway wheels", Wear 265 (2008) 1387-1395

[2] J. R. RICE., "A Path Independent Integral and the Approximate Analysis of Strain Concentration by Notches and Cracks", Journal of Applied Mechanics, vol. 35, pp. 379-386, 1968

[3] Sara Caprioli, ToreVernersson, KazuyukiHanda, Katsuyoshilkeuchi Thermal cracking of railway wheels: Towards experimental validation

[4] Sanders. J. L., "On the Griffith-Irwin Fracture Theory," journal of applied mechanics, vol. 27, No.2, TRANS. MME, Vol. 82, Series E. 1960, pp. 352-353.

[5] Rice, J. R., "The Mechanics of Crack Tip Deformation and Extension by Fatigue," Fatigue Crack Growth, ASTM Spec. Tech. Pub! 415, 1967.

[6] SaraCaprioli, ToreVernersson, KazuyukiHanda, Katsuyoshilkeuchi, "Thermal Cracking of railwaywheels:Towards experimental validation", Tribology International journal, Volume 94, February 2016, Pages 409-420

[7] Johnson KL. "The strength of surfaces in rolling contact Part C: J Mech Eng Sci, 1989;203:15163

[8] Haidari, A., Hosseini-Tehtani, P., " and mechanical loads", Journal of Thermal Stresses, 37: 3450 (2014).

[9] Azadeh Haidari' ParisaHosseini Tehrani," Thermal load effects on fatigue life of a cracked railway wheel", Lat. Am. j. solids struct. vol.12 no.6 Rio de Janeiro June 2015

[10] Chuanxi. S, Jun. Z, Chunyan. W, Xueshan. Z., (2009), "Thermo-Contact Coupling Finite Element Method Analysis of Wheel/Rail Under Sliding Status", International Conference on Information Engineering and Computer Science (ICIECS), Wuhan, China, vol. 1,pp. 1-4, December 19-20.

[11] H. Xu, K. Komvopoulos, "Surface adhesion and hardening effects on elastic-plastic, shakedown and ratcheting behavior of half-spaces subjected to repeated sliding contact", International Journal of Solids and Structures 50 (2013) 876-886

[12] A. F. BOWER, J. Mech. Phw, "cyclic hardening properties of hard-drawn copper and rail steel", Solids Vol. 37. Tie. 4. pp. 45-U70. 1989

[13] S. Courtin, C. Gardin ,G. Be'zine, H. Ben Hadj Hamouda, "Advantages of the J-integral approach for calculating stress intensity factors when using the commercial finite element software ABAQUS", Engineering Fracture Mechanics 72 (2005) 2174-2185.

[14] Shih YS, Chen JJ. The stress intensity factor study of an elliptical cracked shaft. Nucl Engng Des 2002; 214(1-2):137-45. 
[15] Lin XB, Smith RA. Finite element modelling of fatigue crack growth of surface cracked plates. Part I: The numerical technique. Engineerig FractMech 1999;63(5):503-22.

[16] K.O. Findley, S.W. Koh, b, A. Saxena, "J-integral expressions for semi-elliptical cracks in round bars" , International Journal of Fatigue 29 (2007) 822-828

[17] S. Courtin, C. Gardin ', G. Bézine, H. Ben Hadj Hamouda, " Advantages of the $J$-integral approach for calculating stress intensity factors when using the commercial finite element software ABAQUS", Engineering Fracture Mechanics, Volume 72, Issue 14, September 2005, Pages 2174-2185

[18] Majid-Reza Ayatollahi and Sadjad Pirmohammad, "Temperature effects on brittle fracture in cracked asphalt concretes, Structural Engineering and Mechanics, An International Journal, Vol.45, No.1, pp.019-32, 2013.

[19] R. Ghajar, J. Alizadeh K. "Prediction of the subsurface crack growth lifetime in railroad wheel of the Iranian railway system", International Journal of Advanced Design and Manufacturing Technology, Vol. 13, pp. 11-24, 2010

[20] David Y. Jeong Michael E. Carolan Hailing Yu Benjamin Perlman., "fracture mechanics and beam theory analyses of semi-elliptical cracks originating in the base of rail", Proceedings of the 2012 Joint Rail Conference JRC2012 April 17-19, 2012, Philadelphia, Pennsylvania, USA

\section{AUTHORS' BIOGRAPHY}

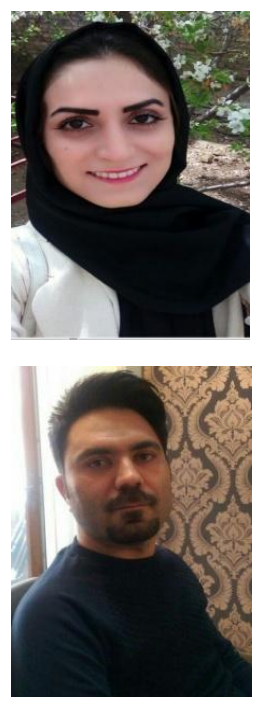

Azadeh Haidari, I am a PhD student in Iran university of science and technology with the field of Applied design and Subcategory of fatigue and fracture mechanics. Most of my studies and articles have been in this field. I have 2 ISI and 1 International conference paper and A book with the subject of fatigue and fracture in railway wheels.

Mojtaba Heidari, He is a graduate student at Isfahan University of Technology, one of the top universities in Iran, who has collaborated with this author in this article. 